\title{
Challenges in chemotherapy delivery: comparison of standard chemotherapy delivery to locoregional vascular mass fluid transfer
}

\author{
Rodney J Lane*,1,2,3, Nyan Y Khin'², Nick Pavlakis ${ }^{5}$, Thomas J Hugh ${ }^{3}$, Stephen J Clarke , \\ John Magnussen ${ }^{2}$, Chris Rogan ${ }^{6}$ \& Roger L Flekser ${ }^{4}$ \\ ${ }^{1}$ Department of Vascular Research, Macquarie University Hospital, NSW, Sydney, Australia \\ ${ }^{2}$ Faculty of Medicine \& Health Sciences, Macquarie University, NSW, Sydney, Australia \\ ${ }^{3}$ Department of Surgery, Royal North Shore Hospital, NSW, Sydney, Australia \\ ${ }^{4}$ AllVascular Pty Ltd, 130-134 Pacific Hwy, St Leonards, NSW 2065, Sydney, Australia \\ ${ }^{5}$ Department of Medical Oncology, Royal North Shore Hospital, NSW, Sydney, Australia \\ ${ }^{6}$ Department of Radiology, Royal Prince Alfred Hospital, NSW, Sydney, Australia \\ * Author for correspondence: Tel.: +61 (02) 9438 4500; rjlane@gmail.com
}

Standard intravenous chemotherapy delivery to neoplasms relies on simple diffusion gradients from the intravascular to the interstitial space. Systemic perfusion creates untoward effects on normal tissue limiting both concentration and exposure times. Regional intra-arterial therapy is limited by drug recirculation and vascular isolation repeatability and does not address the interstitial microenvironment. Barriers to delivery relate to chaotic vascular architecture, heterogeneous fluid flux, increased interstitial and variable solid tumor pressure and ischemia. To address these difficulties, a delivery system was developed allowing mass fluid transfer of chemotherapeutic agents into the interstitium. This implantable, reusable system is comprised of multiple independently steerable balloons and catheters capable of controlling the locoregional hydraulic and oncotic forces across the vascular endothelium.

First draft submitted: 16 October 2017; Accepted for publication: 15 December 2017; Published online: 7 March 2018

Keywords: bulk fluid flux $\bullet$ direct arterial chemotherapy delivery $\bullet$ drug eluding embolic particles $\bullet$ implantable multicatheter vascular access system • isolated hepatic infusion with recirculation and hemofiltration $\bullet$ mass fluid transfer $\bullet$ neoplasms $\bullet$ stop flow techniques $\bullet$ vascular mechanics

The intravenous (iv.) route is the standard pathway of delivering chemotherapy to neoplasms. As cancers have the propensity to disseminate, it is therefore logical to treat all body parts and circulating tumor cells and should always be an integral part of chemotherapy treatment.

Tumors and their draining lymph nodes reside in the interstitial or extravascular space and hence delivery requires the therapy to traverse the vascular endothelium [1]. The prime mechanism is simple diffusion down a concentration gradient aided by an increased intrinsic tumor vascular permeability [2]. The main obstacle is providing sufficient chemotherapy concentration to eradicate the tumor without causing deleterious side effects on normal structures remote from the tumor. The effective concentration of the delivered chemotherapy agent depends upon the relative mass of the organ compared with total body mass in attacking the neoplasm [3]. For example, a pancreatic head tumor may be $35 \mathrm{~g}$ in a $75 \mathrm{~kg}$ patient; this is a relative mass factor of 2000-times while the affected clearing lymph nodes may be 5-7 $\mathrm{mm}$ with a dilution factor of 10,000 to 1 [3]. The concentration limitation also prolongs the time requirement to produce an effective response. Cellular uptake depends on a series of complex biochemical interactions, some active and some passive, but all time dependent. Of equal importance is the desire to reduce the time taken to properly assess a treatment's effectiveness so that therapy can be changed promptly, if necessary. The combination of concentration and time restraints allows tumors to mutate, conjugate or develop mechanisms to mitigate chemotherapeutic actions thereby increasing cancer viability. Side effects may lead to early treatment

Future Medicine 

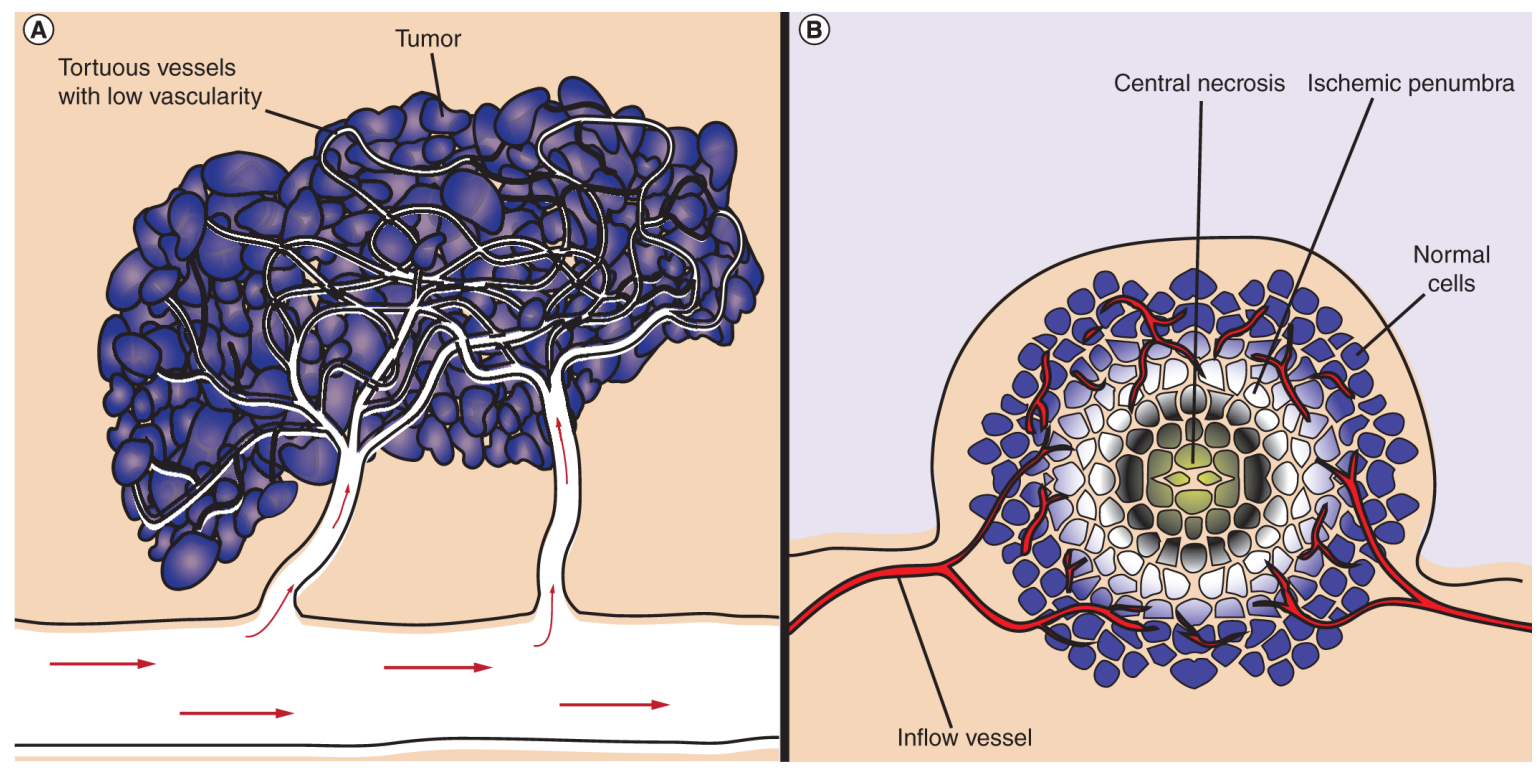

Figure 1. The intravenous route for chemotherapy delivery is impaired by the high resistance vasculature. The tumor vessels are often tortuous, heterogeneous, hypovascular with little or no flow (A). Ischemic apoptosis liberates proteins centrally in the tumor with a resulting high oncotic pressure. This, combined with a decreased functional lymphatic drainage and high vascular permeability, results in an increase in central interstitial fluid pressure, which precludes adequate chemotherapy penetration (B).

cessation and occasional premature death. Further, the intravenous route may also impair the natural immunological response to the tumor via bone marrow suppression and lympholysis.

In addition to the vascular endothelial barriers, the intravenous route for chemotherapy delivery is affected by multiple metabolic and excretory processes including plasma binding [4] as well as the dynamic tumor microenvironment [5]. The tumor vessels are often tortuous, heterogeneous, hypovascular or frequently associated with a low $\mathrm{PO}_{2}$, which can initiate high protein transcription (Figure 1A) [6]. Ischemic apoptosis liberates protein centrally in the tumor with a resulting high oncotic pressure (Figure 1B) [1]. Combined with a decreased functional lymphatic drainage and high vascular permeability, the result is an increase in central interstitial fluid pressure (IFP) precluding adequate chemotherapy penetration $[7,8]$. A further confounding element is the increase in extracellular matrix density from elevated concentrations of collagen, hyaluronan and proteoglycans making the tumor stiffer and creating high pressure gradients [9]. The solid tumor pressure (STP) also relates to unregulated and often unpredictable cellular growth. Rapidly multiplying cells abut, compress and deform their neighboring tissue and may create a pseudo capsule within the host tissue [6]. There also exists a complex interplay between a multitude of physiological constituents including STP, IFP, infusion hydraulics, vascular heterogeneity, endothelial permeability and hypoxia [10-12]. All these erratic variables result in constant 3D spatiotemporal remodeling (Figure 2).

A number of treatment modalities have been proposed in an attempt to overcome these challenges that hinder effective chemotherapy delivery.

\section{Direct arterial delivery/regional chemotherapy infusion}

Part of the solution to increasing the efficacy of dose chemotherapy delivery is to directly infuse the vessels supplying the tumor. Regional infusion encompasses many diverse modalities, including intraperitoneal therapy (for appendiceal and ovarian cancers) [13], intrathecal therapy (for metastatic lesions to the brain) [14] and direct arterial infusion treatment (regional chemotherapy infusion).

One of the best studied areas of regional infusion is that of hepatic arterial infusion (HAI) for secondary colorectal cancer $[15,16]$. Here, patients undergo percutaneous implantation of an indwelling arterial hepatic catheter connected to an infusion reservoir (port-a-cath) or a transcutaneous catheter can be implanted in the gastroduodenal artery and is intermittently connected to an arterial pump thereby delivering chemotherapy continuously over several hours [17]. 


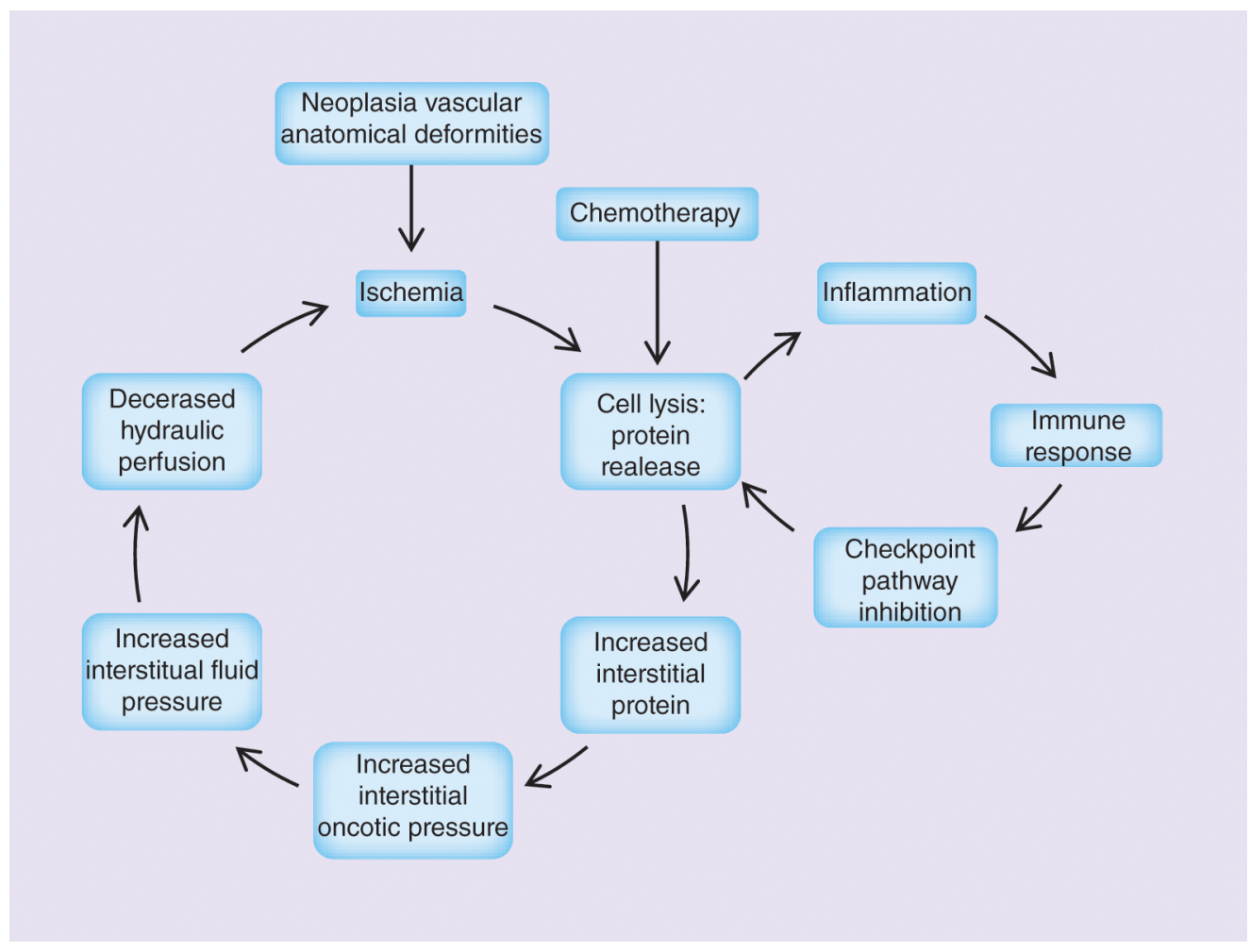

Figure 2. The interactions of anatomical vascular chaos, ischemia, high fluid pressure chemotherapy and immunotherapy are self-propagating. Chemotherapy and ischemia improve antigen exposure inducing inflammatory pathways and hence potentiating checkpoint pathway inhibition. Induced cell lysis releases proteins increasing interstitial fluid pressure and thence initiating hypoperfusion.

The relative advantage (RT) of HAI over the typical intravenous route is given by the following formula:

$$
R T=1+\frac{T B C}{Q(1-e)}
$$

Where the total body clearance (TBC) is calculated by dividing the chemotherapy dose by the area under the time-concentration curve (AUC). Q and e denote the flow past the tumor and the extraction ratio, respectively [3,17]. An important deduction from this equation is that utilizing this treatment allows for a transient increase in local chemotherapy concentration with minimal plasma binding (Figure 3) [18-20]. Therefore, when intravenous and HAI variables are introduced into this equation a relative advantage can be deduced. This has relevance with some compounds that have a very high vascular extraction ratio on first pass removal, such as in fluorouracil derivatives [3]. Although this method has been shown to be modestly beneficial via meta-analysis [3,16], catheter migration and thrombosis [21] as well as the need for laparotomy for device insertion has resulted in limited universal acceptance [22].

\section{Drug eluting embolic particles}

Transcatheter arterial chemoembolization (TACE) involves the delivery of a chemotherapeutic agent, mixed with embolic material, administered selectively into the feeding arteries of the tumor to obtain higher intratumor drug concentrations compared with intravenous therapy, with occlusion of the blood vessel causing infarction and necrosis [23]. Microspheres are the most common embolic agents and can be made from various compounds including glass and resins. These compounds are usually bound to chemotherapeutics. No consensus exists as to the choice of agent [24].

The DC Bead (BTG International Ltd, London, UK) is a novel drug delivery embolization system [25-27].The beads vary in diameter of $100-700 \mu \mathrm{m}$ and are typically hydrophilic, nonreabsorbable hydrogels usually impregnated 


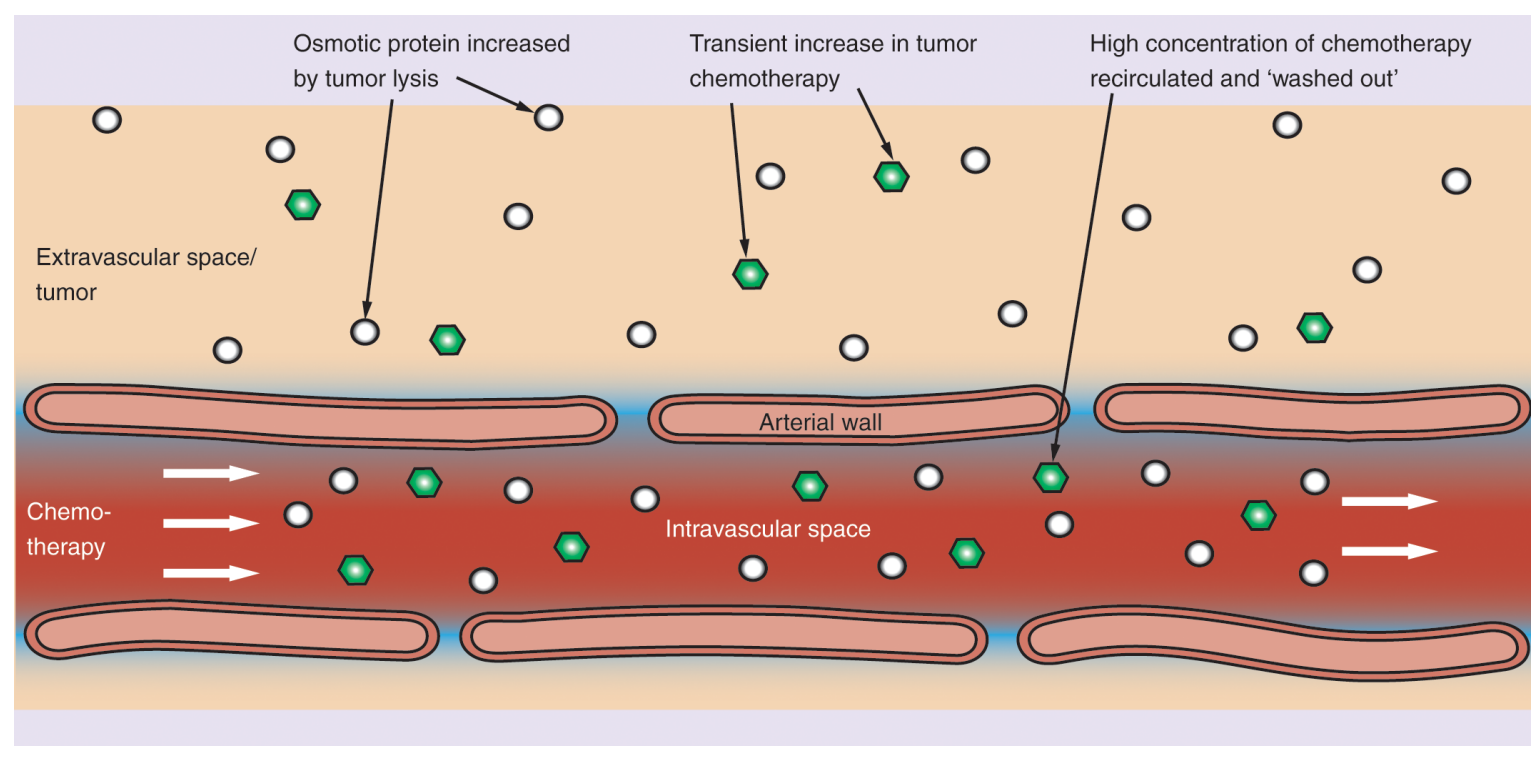

Figure 3. Standard intra-arterial chemotherapy at the tumor vascular interface. The transient increase in concentration of direct intra-arterial chemotherapy is washed out and recirculated. The induced side effects limit both the maximum concentration and area under curve values.

with a chemotherapeutic agent such as doxorubicin [28]. Administration is similar to TACE without the need for lipiodol intermixing $[29,30]$.

The therapeutic advantage of chemo-eluting particles relate to a controlled and sustained locoregional release of therapeutic agents in high concentration with prolonged administration at the targeted tumor thereby reducing its potential systemic toxicity [28]. Rapidly dividing neoplasms often have high vascular density and therefore facilitates self-selecting particle deposition. Embolic ischemia adds to this therapeutic advantage. Doxorubicin beads with TACE deployed in hepatocellular carcinomas have been shown to require fewer treatments compared with conventional TACE and have the same overall survival [31].

The disadvantages relate to occlusion of normal tissue with secondary ischemia. In addition, inadvertent migration of these particles into the systemic circulation can result in pulmonary embolization or infarction [32]. This concept is further complicated by arteriovenous shunts, which are commonly found in tumors [33]. Long-term ischemia induces collateralization related to surrounding endothelial shear stress and hormonal release. This may promote rather than inhibit tumor growth. In addition, the maximum chemotherapy concentration delivered usually remains intravascular and fails to address the problems of chaotic, tortuous and highly resistant vascular beds. In fact, some tumors themselves are hypovascular, which may result in the inability of beads to reach their target [34].

\section{Stop flow techniques}

Cessation of blood flow augments arterial chemotherapy delivery as well as the delivery of immunotherapeutic cancer treatments [35]. The goal of locoregional drug administration is to deliver a higher concentration of chemotherapy to the target compared with the delivery of chemotherapy systemically.

This treatment modality involves deploying an occlusion balloon catheter into the tumor feeding vessel thereby allowing chemotherapy greater time to traverse the vascular endothelium. In Equation 1, as the flow approaches zero and the extraction rate is high, the relative advantage improves dramatically [35]. Several techniques have been developed and include selective arterial infusion [36], celiac axis infusion [37], aortic stop flow infusion [38] and isolated hypoxic infusion. The last two treatment regimes offer the advantage of reducing regional active drug clearance as well as inducing hypoxia of the isolated compartment, which has been shown to increase the cytotoxic action of some anticancer agents, for example, mitomycin $\mathrm{C}$ and doxorubicin [39-41].

In the past, 'isolated' hypoxic abdominal perfusion has been reserved for patients with unresectable pancreatic cancer (stage III/IV) nonresponsive to traditional systemic chemotherapy [42,43]. During abdominal isolated perfusion, balloon catheters positioned within the aorta above the celiac axis and within the vena cava above the hepatic veins stop the blood flow of the abdominal vascular bed creating a virtual 'closed circuit' for perfusion 
of the pancreas, liver and adjacent tissue. Despite leakage from the collateral circulation, a regional concentration advantage of perfused agents has been demonstrated in several studies [44,45]. An additional advantage of hypoxic abdominal perfusion is the decrease in tissue oxygenation and $\mathrm{pH}$. Hypoxia increases the state of tumor cells and inhibits proliferation [40]. Hypoxia also enhances the cytotoxic activity of antiblastic agents such as mitomycin C and doxorubicin. The high incidence of local recurrence postradical pancreatic resection and the pattern of regional metastatic spread makes this technique practical for treating locally advanced pancreatic cancer, which is otherwise very difficult to treat using traditional surgical or medical intervention $[46,47]$.

This technique does have shortcomings including prolonged end organ ischemic time, high hydraulic infusion forces required to adequately perfuse the tumor with chemotherapy as well as the potential genesis of collateral tumor circulation. Confounding deficiencies in this technique can occur during balloon deflation, which may result in the rapid exit of unwanted residual chemotherapeutic agents into the venous circulation [48]. Inability to repeat treatment and difficulty related to radiographic vascular superselection has resulted in a limited number of clinicians using this treatment modality.

\section{Isolated \& percutaneous hepatic infusion with recirculation \& hemofiltration}

Hepatic perfusion with high-dose chemotherapy directly into the liver via an open-surgical procedure, known as isolated hepatic perfusion (IHP), has been demonstrated to control disease and extend survival for patients with unresectable hepatic metastases $[49,50]$. It takes approximately 8 hours to complete and the recovery period is protracted with patients spending 2-3 days in an intensive care unit immediately after the procedure followed by an additional $10-15$ days in the hospital prior to discharge. IHP has not been widely adopted as a treatment because it is an open-surgical procedure that is limited to one-time-only treatment and is associated with a high morbidity and mortality [51].

Percutaneous hepatic perfusion was developed to replace IHP. This technique utilizes a double-balloon catheter system (produced by Delcath Systems, NY, USA) positioned percutaneously in the retrohepatic vena cava [52-54]. The double-balloon catheter has a unique construction with a large central lumen, three accessory lumina and fenestrations throughout its length that allows the hepatic venous effluent to be isolated and filtered through an extracorporeal filtration system. The two balloons on either end of the catheter are positioned inferior and superior to the hepatic veins $[55,56]$. The venous outflow from the liver is filtered through this double-balloon catheter into the extracorporeal filtration system. Filtered blood is then returned to the systemic circulation through a catheter in the internal jugular or subclavian veins. Cannulation of the femoral artery with placement of a catheter into the proper hepatic artery allows for directed infusion of chemotherapy into the organ containing the pathologic tissue [57,58]. Once the extracorporeal circuit is established, chemotherapy can be delivered via the hepatic artery with simultaneous extracorporeal blood filtration via the inferior vena cava and systemic return by way of the neck veins [59].

Although initial results have been encouraging, numerous difficulties have been identified, namely, hematological, cardiovascular, hepatic, nephrotic and gastrointestinal toxicity [52,54,60]. Surgical morbidity, cost and repeatability are additional significant drawbacks. Also, this technique does not address the all-important tumor microenvironment.

\section{Simple diffusion versus mass fluid transfer \& bulk fluid flux}

The delivery systems described have been unable to address the 3D spatiotemporal barriers that exist in the delivery of chemotherapy. In conventional chemotherapy delivery, the maximum concentration remains intravascular. However, cancer cells reside within the extravascular space and therefore therapy is required to traverse their semipermeable membranes to attain their maximum therapeutic concentration. The laws that govern basic diffusion were described by Fick [61]. The major variables of diffusion are solute concentration, time of exposure and crosssectional vascular area. In the chemotherapeutic setting, the derived pharmacokinetic variables are of maximum concentration $\left(\mathrm{C}_{\max }\right)$ and an estimate of exposure is described by the AUC. These values are important for describing response curves and potential side effects of chemotherapeutic agents. Darcy's Law expands on these principles adding blood viscosity and infusion velocity as further variables governing fluid flux across these membranes and hence the possible advantage of bolus delivery of therapeutic agents [62].

Mass fluid transfer can be defined as the delivery of soluble molecules across a semipermeable membrane while bulk fluid flux is the process used by lipid insoluble proteins to cross the capillary endothelium $[7,8]$. The latter has relevance to local antibody delivery to solid neoplasia. Bulk fluid flux is defined by Starling's law: "fluid movement due to filtration across the wall of a capillary is dependent on the balance between the hydrostatic pressure gradient 
$(\Delta \mathrm{P})$ and the oncotic pressure gradient across the capillary $\left(\Delta \prod\right)$ " $[7,8,63]$. Starling's equation is as follows:

$$
J_{v}=K_{f}\left(\left(P_{c}-P_{i}\right)-\sigma\left(\Pi_{c}-\Pi_{i}\right)\right)
$$

(Equation 2)

1. Where $\mathrm{J}_{\mathrm{v}}$ is the transendothelial solvent filtration in volumes per second;

2. $\mathrm{K}_{\mathrm{f}}$ is the filtration constant that relates to the hydraulic conductance and capillary surface area;

3. $\mathrm{P}_{\mathrm{c}}$ is the capillary pressure;

4. $\mathrm{P}_{\mathrm{i}}$ is the interstitial pressure;

5. $\sigma$ is the reflection co-efficient related to the re-absorption of albumen;

6. $\prod_{i}$ is the oncotic interstitial pressure; and

7. $\prod_{c}$ is the capillary oncotic pressure.

In the delivery of chemotherapeutic agents to solid tumors, the filtration constant can be manipulated by changing $\Delta \mathrm{P}\left(\mathrm{P}_{\mathrm{c}}-\mathrm{P}_{\mathrm{i}}\right)$ and also $\Delta \prod\left(\prod_{\mathrm{c}}-\prod_{\mathrm{i}}\right)$. One way to improve $\Delta \mathrm{P}_{\mathrm{i}}$ is to increase the infusion pressure above normal cardiac infusion pressures. To improve $\Delta \prod$, the intravascular oncotic pressure can be reduced optimally by decreasing the plasma protein concentration. $\mathrm{K}_{\mathrm{f}}$ can be improved by physical factors such as hyperthermia and ischemia [64]. Venous end capillary bed reabsorption of chemotherapy can be manipulated by increasing venous pressure.

Starling's equation can therefore be summarized in chemotherapy delivery as the flux across the tumor circulation. The focus of chemotherapy delivery is therefore dependent on three central concepts, in other words, inflow hypertension, removing plasma proteins and obstructing venous outflow [65].

\section{Vascular mechanics}

To remove blood and plasma proteins locoregionally from the tumor, the inflow pressure must be reduced so that there is no discernible flow. This requires pressure to be reduced below critical closing pressure $(20 \mathrm{mmHg}$ at capillary level) [66]. Achieving this allows plasma proteins and blood to be washed out into the venous system with saline. The net effect is an outward oncotic flux of $30 \mathrm{mmHg}$.

The concept of generalized hypoproteinemia and interstitial edema is well known in clinical equivalents such as Kwashiorkor and nephrotic syndrome. The mechanical requirement to reduce inflow below critical closing pressure requires occlusion catheters and balloons usually placed superselectively to occlude not only the axial inflow system but also collateral flow. This important step needs to be modulated and regulated by continuous pressure transduction assessment. Once the oncotically active components are removed, the venous outflow can then be occluded via various means such as positive end expiratory pressure (PEEP) or balloon occlusion of the hepatic veins via catheterization of the internal jugular vein. PEEP has the added advantage over selective venous occlusion as it results in a generalized increase in venous pressure thus avoiding shunting of the venous flow from one segment of the organ to the other.

Venous outflow obstruction ensures retention of chemotherapy in the interstitial space. Therapeutic agents can then be infused at suprasystolic pressures to overcome elevated vascular resistance intrinsic to many tumors. Most importantly the measured chemotherapy infusion pressure is designed to remain less than the measured outflow venous pressure to avoid systemic contamination (Figure 4).

There are a multitude of intrinsic physical factors that can also affect membrane permeability. One such factor is ischemia or oncosis, which plays an integral part of the mass fluid transfer concept [67]. Others include hyperthermia, $\mathrm{pH}$ and osmolarity variation all of which may assist in the therapeutic delivery of substances across the neoplastic cells semipermeable membranes.

\section{Hyperperfusion}

Of paramount importance to cellular chemotherapy absorption is the concept of 'cellular hyperperfusion' which is defined as an increase in inflow pressure beyond the pressure that can be attained from the normal cardiac cycle. In the past, the technique of limb hyperperfusion has been successfully utilized to overcome high peripheral resistance in critical limb ischemia [68-70]. Figure 5 shows thermographic images of a gangrenous foot hyperperfused at $150 \%$ of the normal inflow pressures throughout the cardiac cycle [71,72]. The same basic hardware was modified to hyperperfuse solid tumors with chemotherapy. 


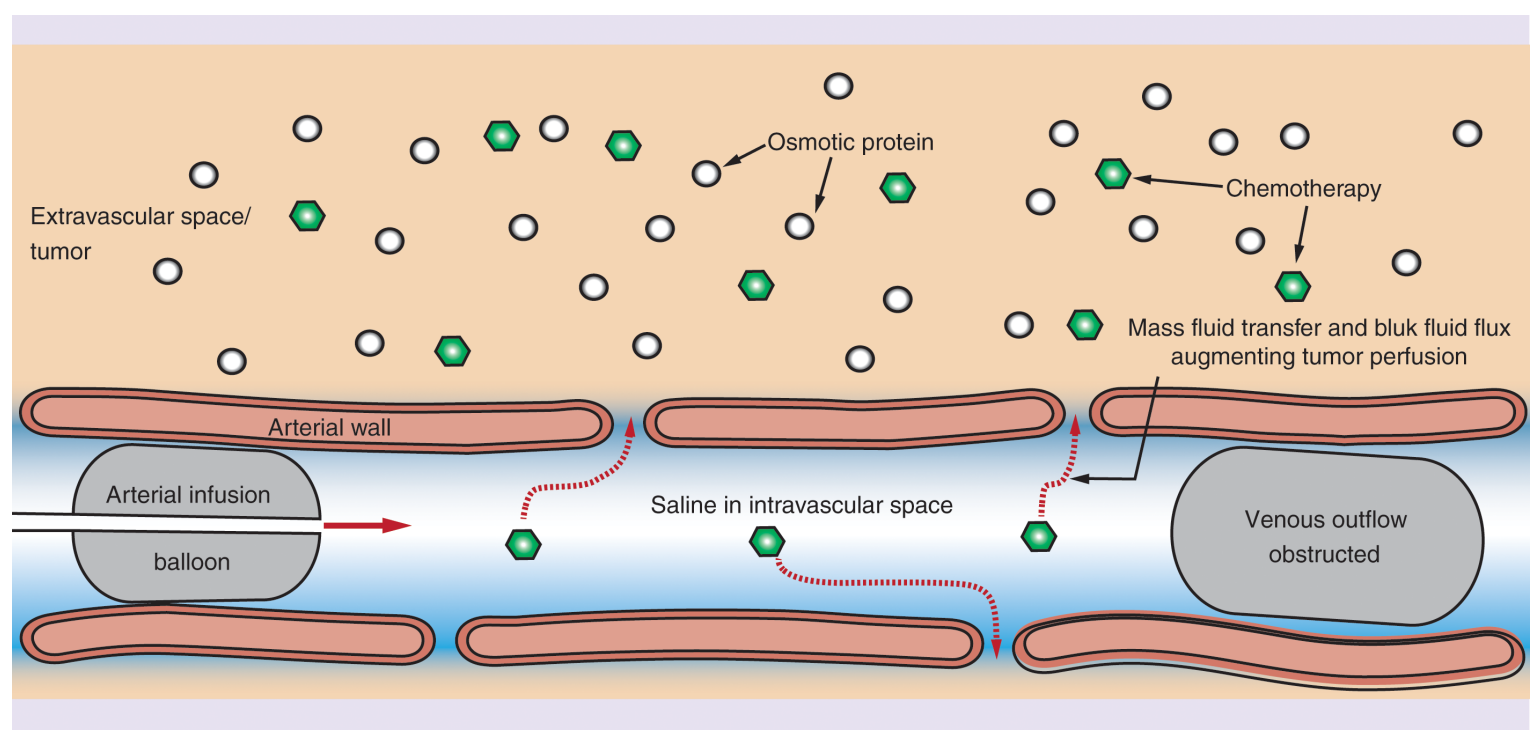

Figure 4. Mechanisms of vascular isolation and mass fluid transfer. To remove blood and plasma proteins locoregionally from the tumor, the inflow pressure must be reduced so that there is no discernible flow (vascular isolation). This requires intravascular pressures to be reduced below critical closing pressure (20 $\mathrm{mmHg}$ at capillary level). Achieving this allows plasma proteins and blood to be washed out into the venous system with saline. The net effect is therefore an outward chemo-oncotic flux of $30 \mathrm{mmHg}$. It is this chemo-oncotic flux that ensures that delivery of chemotherapy to the tumor across the vascular semipermeable membrane (mass fluid transfer) is augmented. The mechanical requirement to reduce inflow below critical closing pressure requires occlusion catheters and balloons usually placed superselectively. Venous outflow can then be occluded via various means such as positive end expiratory pressure or balloon occlusion.

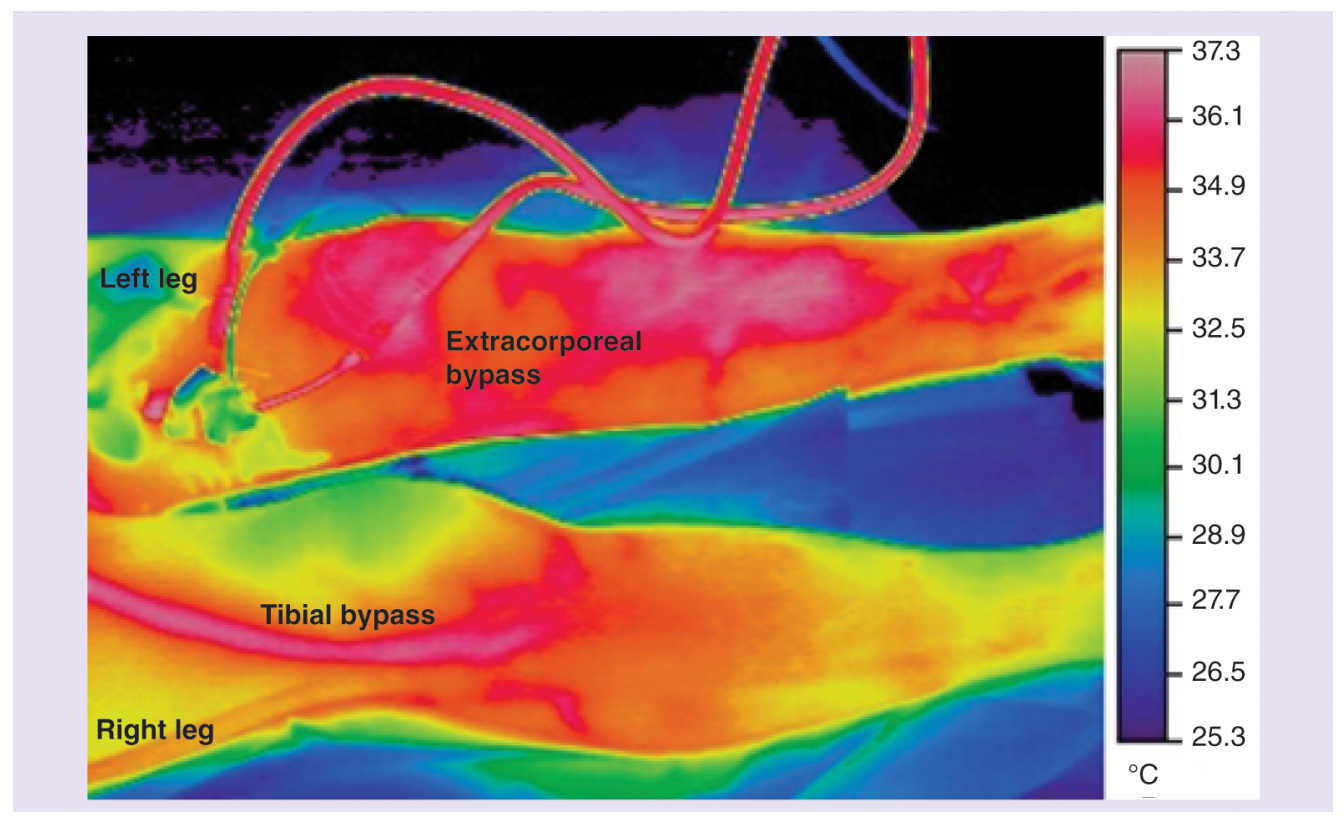

Figure 5. Thermographic images of a gangrenous foot hyperperfused at $150 \%$ of the normal inflow pressures throughout the cardiac cycle. Gangrenous left leg hyperperfused with extracorporeal bypass. Outcomes were shown to be superior to standard to tibial bypass (right leg).

\section{Multicatheter access system}

To optimize cellular endothelial permeability, mass fluid flow as well as hydraulic and oncotic forces, an implantable multicatheter vascular access system was developed (AllVascular Pty Ltd, St Leonards, Australia). The system's 


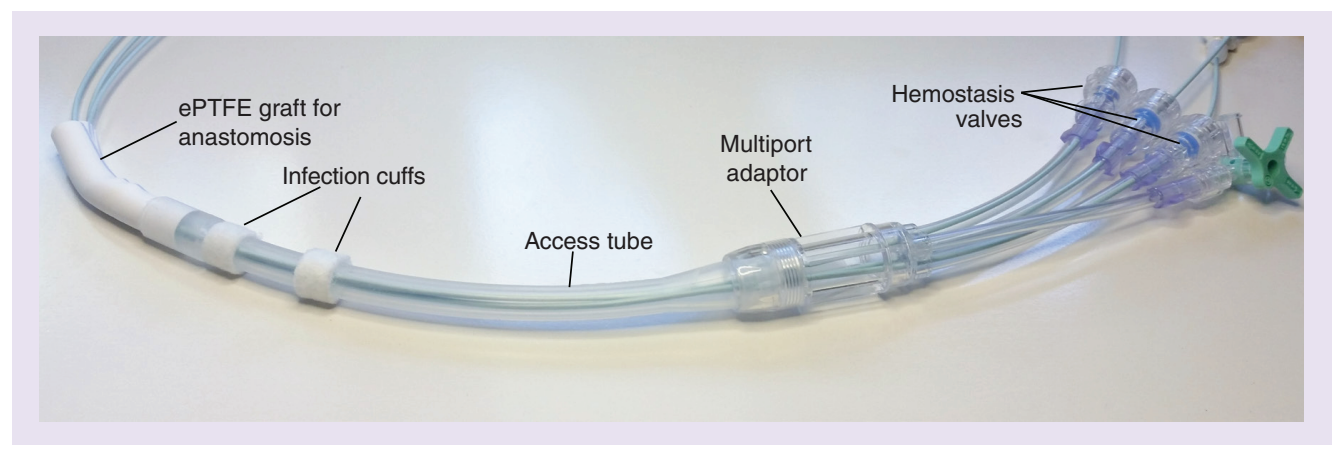

Figure 6. Implantable multicatheter access system.

prerequisites for mass fluid transfer are an implantable, tunneled, transcutaneous large bore port. In the dormant phase, the device is plugged with a disposable flexible plunger with a chamfered tip.

This abuts the anastomosis to create a continuous interface with the vascular endothelium and maximizes laminar flow. The plunger shaft is perforated allowing for anticoagulation of the potential space between the plunger and the internal surface of the silicone port. These features minimize thromboembolic complications. To activate the system, the plunger is removed and the access system is connected to a multiport adaptor which can accommodate up to six cannulas (Figure 6). Each port on the adaptor is equipped with a hemostasis valve to provide a seal to avoid blood loss when introducing catheters.

Superselecting target vessels using this system are made possible with the aid of radiological guidance. The system design enables carefully modulated pressure control via pressure transduction. Importantly the delivery process is easily repeatable and is controlled from a single point source. Access is possible to both the primary and secondary tumors either simultaneously or individually. Additionally, the device has been developed to minimize potential complications. The implantable access tube is silicone, minimizing tissue reaction and flexible to accommodate independent catheter steerage. The ePTFE in the anastomosis is resilient and difficult to perforate with guide wires. The arterial anastomosis is double layered and the two suture lines are offset to minimize guidewire perforation, hemorrhage and false aneurysms. It is also designed to withstand elevated suprasystolic pressures. Polyester felt placed circumferentially around the silicone access tube serves as an infection barrier and promotes dense fibrous ingrowth, minimizing inadvertent patient dislodgement (Figure 6).

Potential complications relate to multiple catheter deployments remote from the device, including dissection and thromboembolism.

\section{Pilot study system deployment}

The safety and feasibility of a multicatheter access system has previously been assessed in a pilot study involving patients with secondary colorectal liver tumors [73]. A total of 57 infusions were delivered across a total of ten patients each treated over a 4-week interval [73]. To obtain capillary closing pressure (normally $20 \mathrm{mmHg}$ at the precapillary level), balloon catheter control of the celiac axis, superior mesenteric axis as well as hepatic artery isolation at two levels was required (Figure 7). In addition, the venous outflow was controlled by PEEP which ensured pressures in excess of $30 \mathrm{mmHg}$ within the liver were sustained during bolus infusion of chemotherapy delivery. The tumor was subsequently washed out with saline and infused with a chemotherapy solution. This method gives an oncotic gradient of $30 \mathrm{mmHg}$ into the interstitial space, which may be supplemented by a high protein concentration contents within the tumor.

To minimize rapid re-absorption of fluid and chemotherapy from re-entering the systemic circulation, the induced venous hypertension was maintained for several minutes after the gradual arterial inflow was reconstituted. Some systemic washout was inevitable once the balloon inflation was released. In the pilot study, evidence of this systemic recirculation was confirmed by the accumulation of platinum levels after an infusion had been completed [73].

The results confirmed chemotherapy infusions with hepatic vascular isolation can be achieved with targeted selectivity and minimal complications using this implantable multicatheter access system. Biweekly administration of chemotherapy and compressing treatment time into 1 month minimizes the time for the tumor to mutate or introduce mechanisms to mitigate chemotherapy effectiveness and allows patients to promptly return to normal 


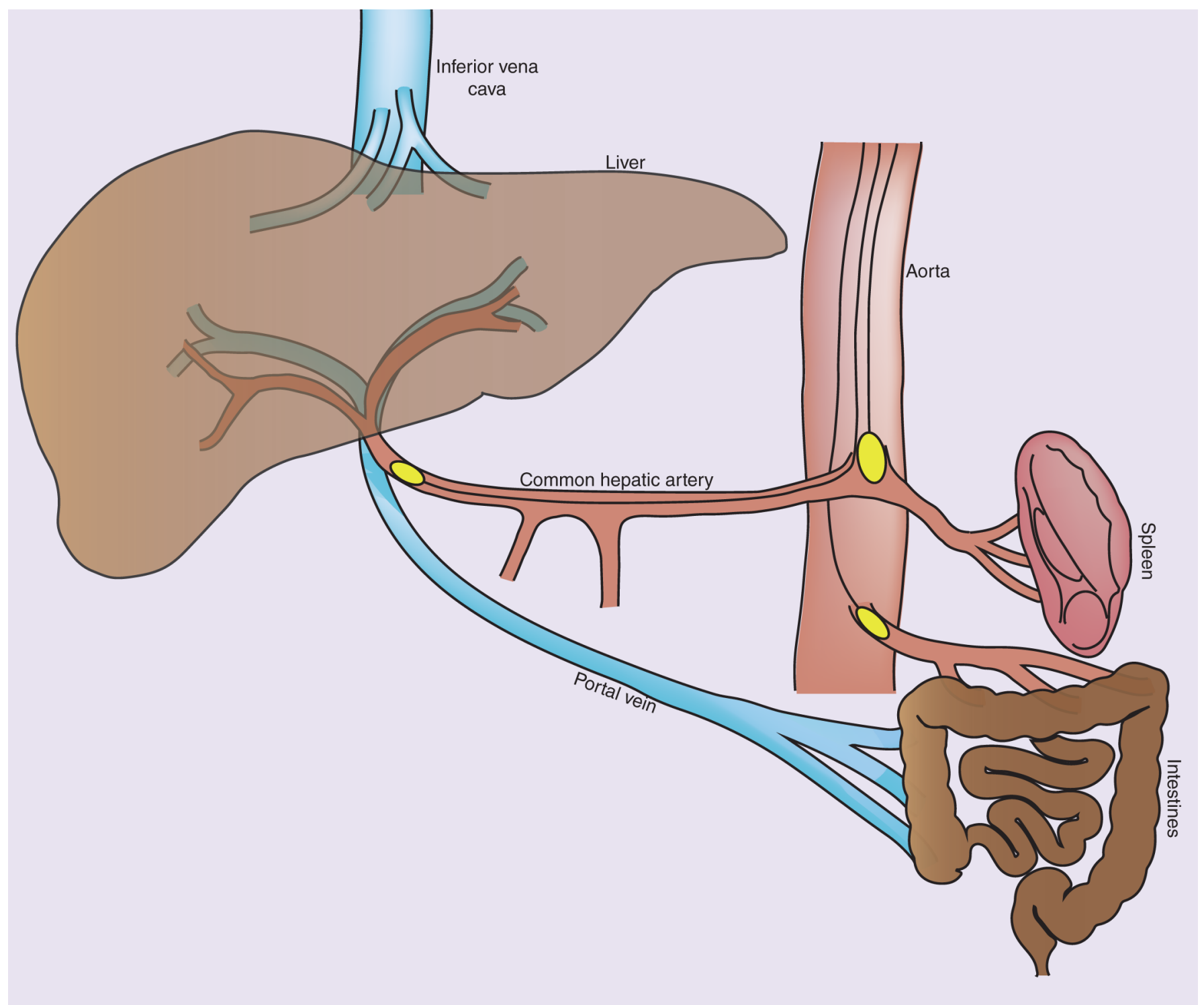

Figure 7. A schematic of the superselection of the hepatic artery for targeted chemotherapy delivery while simultaneously occluding the remaining branches of the celiac axis and inferior mesenteric artery.

life [73]. This time compression also allows the oncologist to assess progression/regression and modify therapy technique.

\section{Direct comparison of mass fluid transfer with routine systemic intravenous therapy}

A typical patient infused with systemic iv. oxaliplatin of $85 \mathrm{mg} / \mathrm{m}^{2}$ over 2 hours can be expected to produce an unbound (active) $\mathrm{C}_{\max }$ of $0.69 \mu \mathrm{g} / \mathrm{mL}$ and an AUC of $4 \mu \mathrm{g} / \mathrm{mL} / \mathrm{h}$ [73]. In contrast should a clinician utilize the hepatic closed segment isolation system with mass fluid transfer, one can expect a local unbound (active) $\mathrm{C}_{\max }$ of $750 \mu \mathrm{g} / \mathrm{mL}$ and an AUC of $300 \mu \mathrm{g} / \mathrm{mL} / \mathrm{h}$ in addition to the known $4 \mu \mathrm{g} / \mathrm{mL} / \mathrm{h}$ with recirculation [73]. As the closed segment system relies on artificially high venous pressures supported by elevated inflow hyperperfusion pressures, the resultant chemotherapy exposure time is high.

Figure 8 summarizes some of the obstacles in delivering intravenous chemotherapy to target cells. Vascular isolation liver therapy allows the dilution factor to be reduced to the mass of the target organ compared with total body mass. This approach minimizes the perfusion of unwanted effects on sensitive organs. Checkpoint immune inhibition is maximal locoregionally [74]. As the plasma proteins are removed locally before infusions, the plasma binding of active agents is minimized; oxaliplatin is $85 \%$ rapidly and irreversibly inactivated by plasma proteins [75]. 


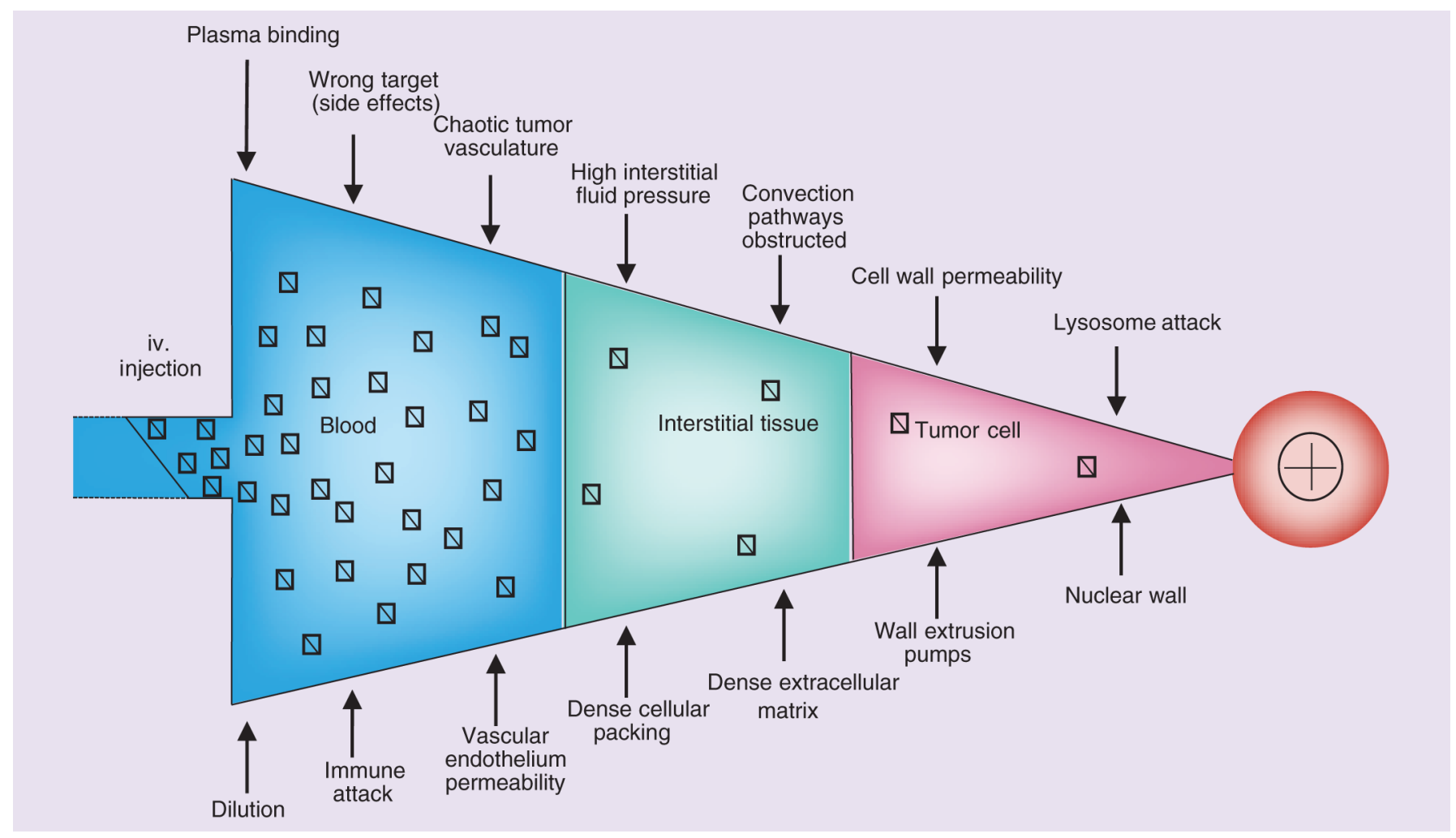

Figure 8. Standard intravenous chemotherapy delivery to neoplasms relies on simple diffusion gradients from the intravascular to the interstitial space. Summarizes numerous barriers which prevent efficient iv. chemotherapy delivery to the target tumor cell.

iv.: Intravenous

Removing the intravascular oncological active plasma protein creates an intravascular to extravascular transfer gradient of approximately $30 \mathrm{mmHg}$. This is then augmented by the higher protein concentrations often in the tumor interstitium $[7,8]$. High pressure bolus injection (Darcy's Law) improves the vascular endothelium mass fluid flux to counter elevated vascular resistance associated with the chaotic vascular architecture. The local permeability is improved by the synergistic effects of the elevated hydraulic and oncotic gradients. The induced interstitial edema may also improve the dense cellular packing and convection pathways [9,62]. Local tumor defences, including lysosome engulfment, are concentration dependent and may be neutralized by overwhelming intracellular chemotherapy gradients $[9,62]$. However, these optimal hemodynamics may have minimal effect on the genetic capability of tumors to mutate and become multidrug resistance.

After resumption of normal flow when the catheters are removed, the high vascular tumor resistance and increased interstitial edema renders intravascular reabsorption of chemotherapy difficult. This combined with a known tumor hypoplastic lymphatic system results in the chemotherapy now 'locked in'. Increased lymphatic flow through surrounding normal lymphatics may be helpful in treating lymph node deposits.

\section{Direct comparison of mass fluid transfer with hepatic arterial infusion}

HAI takes advantage of the fact that a proportion of the arterial blood volume delivered to the liver and particular liver tumors is via the hepatic artery [76]. There is therefore a transient increase in hepatic artery chemotherapy concentration during activation of the forward flow chemotherapy perfusion pump. This concentration increase is limited by recirculation. Confounding chemotherapeutic factors include an immediate chemotherapy dilution as blood flow within the artery reaches $300 \mathrm{~mL} / \mathrm{min}$ within the hepatic artery and $1200 \mathrm{~mL} / \mathrm{min}$ while transversing the portal system intrahepatically. In total, $15 \%$ of the tumor vascular supply is by the portal system.

The hepatic chemotherapy mass fluid transfer method requires intermittent obstruction of both portal and hepatic arterial inflows with multiple catheters selected segmentally, lobar or the liver in its entirety. Plasma proteins are subsequently removed allowing the transfer of chemotherapy to the interstitium of the tumor. This is achieved due to the elevated oncotic gradient that exists between the intravascular and extracellular compartments. During 
infusion venous outflows are obstructed minimizing systemic contamination. Chemotherapy is delivered as a bolus to its intended target at suprasystolic pressures. Reduction of the portal flow activates the hepatic artery buffer response increasing global hepatic arterial blood flow and also shunts chemotherapy into the portal system via the peribiliary anastomosis [76].

A method of addressing the $15 \%$ of blood supply to the tumor by the portal system involves balloon occlusion and perfusion of the splenic artery at the splenic hilum. As the spleen is an end organ, chemotherapy delivered into the splenic hilum results in indirect perfusion of the portal system via the splenic vein. This method is repeatable.

\section{Relevance of mass fluid transfer to in vitro testing}

The known safe peak dose of unbound oxaliplatin delivery at $130 \mathrm{mg} / \mathrm{m}^{2}$ is about $0.9 \mu \mathrm{g} / \mathrm{mL}$ [77]. In comparison, a concentration of $37 \mu \mathrm{g} / \mathrm{mL}$ (67.5 micromoles) of oxaliplatin is the lethal dose required to kill $99.9 \%\left(\mathrm{LD}_{99.9}\right)$ for squamous cells (SM1573) [78]. This can only be achieved safely with locoregional treatment. The relative sensitivity of the tumor to therapy may be more predictable, as delivery capabilities become more objective. Local environment manipulation may also be easier to achieve and therefore becomes less unpredictable. In the above example, heating to $41^{\circ} \mathrm{C}$ improves the $\mathrm{LD}_{99.9}$ by $180 \%$. Local measures to combat increased solid intratumor pressure, such as hyaluronidase, collagenase and abraxane delivery may also be efficacious.

\section{Future perspective: direct comparison of mass fluid transfer with previous locoregional approaches}

The breast

Many of the principles of locoregional delivery of chemotherapy were incorporated by Stephens in stage III breast cancer treatment $[79,80]$. Intra-arterial infusion with superselection was possible from the groin and localization of the tumor blood supply was achieved with selective blue dye infusion. The results were impressive but the side effects due to chemotherapy recirculation were extensive. Suggested improvements would include the use of a long balloon to occlude all the collateral arteries that are not involved in direct arterial supply to the tumor (Figure 9A).

Plasma protein washout is possible before venous outflow is occluded via a single long balloon occluding the internal and lateral thoracic and pectoral veins supplemented by PEEP. This maneuver also minimizes intercostal perforating venous outflow (Figure 9B). The essence of the locoregional isolation technique allows the clinician to manipulate the tumor microenvironment. A simple needle into the breast allows measurement of local chemotherapy concentration and interstitial pressure surrounding the tumor. The interstitial chemotherapy dilution may indicate local lymphatic perfusion.

\section{Mass fluid transfer has the potential to treat most solid tumors}

Immune-checkpoint modifiers offer a broad and diverse opportunity to enhance antitumor immunity with the potential to produce promising clinical responses [81-83]. However, local tumor inflammation is needed to precipitate the activation of the checkpoint inhibitory pathway (Figure 2) [84].

Multicatheter local vascular isolation of organs may be helpful in initiating inflammation by triggering tumor lysis. Ischemia, elevated intrafluid pressure and high-concentration chemotherapy may combine to create a local aggressive immune response and hence a better response to locally delivered checkpoint inhibition (Figure 2). The advantage of delivering these checkpoint modifiers locally minimizes the potential for more serious systemic autoimmune complications.

Although mass fluid transfer has the potential to treat most solid tumors. Several tumors, such as brain and pancreatic tumors, present unique physical and biochemical barriers that will need to be overcome. Brain capillary endothelial cells are interconnected by tight junctions, with limited fenestrations and pinocytotic vesicles that form a barrier to prevent unimpeded diffusion into the brain. Therefore, progress in treating this disease may be dependent on the disruption of this blood-brain barrier. Also, given the number of agents that are substrates for active efflux at the blood-brain barrier there will need to be a modification of drug structures to diminish efflux transporter affinity and perhaps co-administration of transport inhibitors are required to enhance delivery of anticancer drugs. Pancreatic tumors harbor a dense, desmoplastic stroma that serves to limit the delivery of chemotherapeutic agents to these tumors. Efficacious delivery of chemotherapy to these tumors via mass fluid transfer requires further investigation. 


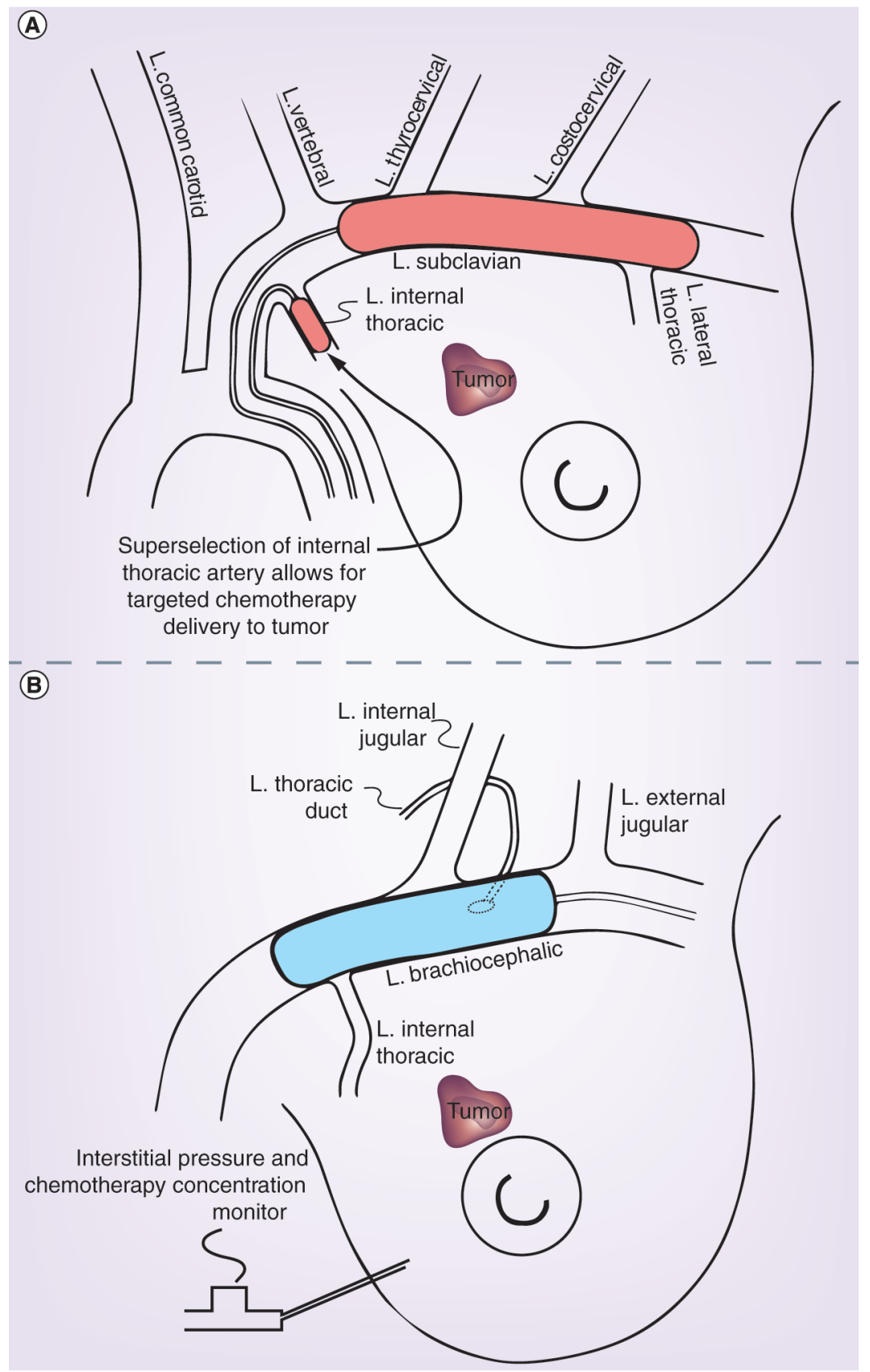

Figure 9. Locoregional intra-arterial breast chemotherapy delivery (A) with venous outflow obstruction (B).

\section{Interaction with new technology}

The US FDA has recently approved fluciclovine F18 for PET imaging in males with suspected prostate carcinoma recurrence based on PSA elevation $[85,86]$. Prostate arterial isolation has been shown to be safe and efficacious for benign prostatic hypertrophy [87]. The use of bulk fluid flux and multicatheter isolation may prove helpful in the focal treatment of pelvic recurrence in an otherwise difficult area to treat.

\section{Conclusion}

The current paradigm to treat solid neoplasia using systemic treatment alone encounters intrinsic difficulties related to the perfusion penetration and permeability of chemotherapy. The barriers relate to the chaotic tumor vasculature and elevated pressure within the tumor. Mass fluid transfer and bulk fluid flux combines intravenous, intra-arterial, stop flow and superselective locoregional chemotherapy delivery. The technique adds venous outflow obstruction 
for chemotherapy retention and locoregional hypertension for added penetration and intravascular protein removal. Most importantly, the system is repeatable, measurable and addresses the tumor microenvironment. The essential components needed to manipulate mass fluid transfer and bulk fluid flux are therefore inherently dependent on specifically designed vascular hardware.

Executive summary

\section{Background}

- The standard treatment of solid organ neoplasms relies on the systemic delivery of intravenous chemotherapy.

- Effective treatment relies on the administration of a sufficient chemotherapy concentration to eradicate the tumor without causing deleterious side effects on normal structures remote from the tumor.

- In an attempt to overcome the challenges of effective chemotherapy delivery a number of treatment modalities have been proposed:

1. Direct arterial delivery

2. Drug eluting particles

3. Stop flow techniques

4. Isolated hepatic infusion with recirculation and hemofiltration

5. Implantable multicatheter access system

Simple diffusion versus mass fluid transfer \& bulk fluid flux

- Mass fluid transfer can be defined as the delivery of soluble molecules across a semipermeable membrane.

- Bulk fluid flux is the process used by lipid insoluble proteins to cross the capillary endothelium.

Vascular mechanics

- Optimal delivery of chemotherapy is dependent on locoregional control of the tumor microenvironment.

- Vascular inflow pressure is required to be below critical closing pressure.

- Venous outflow obstruction ensures retention of chemotherapy in the interstitial space.

- Therapeutic agents can be infused at suprasystolic pressures to overcome elevated vascular resistance intrinsic to many tumors.

Multicatheter access system

- An implantable vascular access system with the capacity for multiple independently steerable targeting catheters.

- The system allows for repeatable vascular isolation that optimizes endothelial permeability and bulk fluid flow.

Direct comparison of mass fluid transfer with routine systemic intravenous therapy

- Locoregional delivery enables an improvement of 2-3 orders of magnitude compared with intravenous therapy. Direct comparison of mass fluid transfer with hepatic artery infusion

- Hepatic artery infusion results in a transient increase in hepatic artery chemotherapy concentration during activation of the forward flow chemotherapy perfusion pump.

- The hepatic mass fluid transfer method requires intermittent obstruction of both portal and hepatic arterial inflows with multiple catheters.

- Plasma proteins are removed allowing the transfer of chemotherapy to the interstitium of the tumor.

- Obstruction of venous outflows prevents systemic contamination and therefore chemotherapy is delivered as a bolus to its intended target at suprasystolic pressures.

Relevance of mass fluid transfer to in vitro testing

- The relative sensitivity of the tumor to therapy may be more predictable, as delivery capabilities become reliable, for example, squamous cells SM1573 have a $\mathrm{LD}_{99.9}$ of $37 \mu \mathrm{g} / \mathrm{mL}$ (67.5 micromoles) of oxaliplatin. This can only be considered possible by local treatments.

- In the above example, heating to $41^{\circ} \mathrm{C}$ improves the $\mathrm{LD}_{99.9}$ by $180 \%$.

Direct comparison of mass fluid transfer with previous locoregional approaches

- In the previous locoregional treatment of stage III breast cancer, superselection and intra-arterial chemotherapy achieved good results with severe side effects.

- The repeatable vascular hardware system allows manipulation of inflow, outflow and oncotic gradients minimizing systemic chemotherapy contamination.

- A simple needle into the breast allows measurement of local chemotherapy concentration and interstitial pressure within the tumor. The interstitial chemotherapy dilution may indicate local lymphatic perfusion.

Mass fluid transfer has the potential to treat most solid tumors

- Multicatheter local vascular isolation of organs may be helpful in initiating inflammation by triggering tumor lysis.

- Ischemia, elevated interstitial fluid pressure and high-concentration chemotherapy may combine to create a local aggressive immune response and hence a better response to locally delivered checkpoint inhibition.

Interaction with new technology

- The use of bulk fluid flux and multicatheter isolation may prove helpful in the focal treatment of pelvic recurrence of prostate cancer with the recent approval by the US FDA of fluciclovine F18. 


\section{Acknowledgements}

The authors would like to thank the New South Wales Medical Devices Fund for making this research possible.

Financial \& competing interests disclosure

RJ Lane is a Shareholder and Scientific Advisor for Allvascular Pty Ltd but receives no financial remuneration. R Flekser is a Scientific Registrar employed and subsidized by Allvascular Pty Ltd. NY Khin is a PhD student subsidized by AllVascular Pty Ltd. The authors have no other relevant affiliations or financial involvement with any organization or entity with a financial interest in or financial conflict with the subject matter or materials discussed in the manuscript apart from those disclosed.

No writing assistance was utilized in the production of this manuscript.

\section{Open access}

This work is licensed under the Attribution-NonCommercial-NoDerivatives 4.0 Unported License. To view a copy of this license, visit http://creativecommons.org/licenses/by-nc-nd/4.0/

\section{References}

Papers of special note have been highlighted as: $\bullet$ of interest; $\bullet \bullet$ of considerable interest

1. Rofstad EK, Galappathi K, Mathiesen BS. Tumor interstitial fluid pressure - a link between tumor hypoxia, microvascular density, and lymph node metastasis. Neoplasia 16(7), 586-594 (2014).

2. Albini A, Sporn MB. The tumour microenvironment as a target for chemoprevention. Nat. Rev. Cancer 7(2), 139-147 (2007).

3. Muchmore JH, Wanebo HJ. Regional chemotherapy: overview. Surg. Oncol. Clin. N. Am. 17(4), 709-730, vii (2008).

- Provides an overview of current regional chemotherapy practices and the important contribution these techniques can have on the development of newer therapies for advanced solid malignancies.

4. Smith DA, Di L, Kerns EH. The effect of plasma protein binding on in vivo efficacy: misconceptions in drug discovery. Nat. Rev. Drug Discov. 9, 929-939 (2010).

5. Pattabiraman DR, Weinberg RA. Tackling the cancer stem cells - what challenges do they pose? Nat. Rev. Drug Discov. 13, 497-512 (2014).

6. Ariffin AB, Forde PF, Jahangeer S, Soden DM, Hinchion J. Releasing pressure in tumors: what do we know so far and where do we go from here? A review. Cancer Res. 74(10), 2655-2662 (2014).

7. Stapleton S, Milosevic MF. Pressure gradients in solid tumors. In: Cancer Targeted Drug Delivery - An Elusive Dream. Bae YH, Mrsny RJ, Park K (Eds). Springer-Verlag, NY, USA, 241-272 (2013).

8. Wragg JW, Bicknell R. Vascular targeting approaches to treat cancer. In: Cancer Targeted Drug Delivery - An Elusive Dream. Bae YH, Mrsny RJ, Park K (Eds). Springer-Verlag, NY, USA, 59-95 (2013).

9. Cox TR, Erler JT. Remodeling and homeostasis of the extracellular matrix: implications for fibrotic diseases and cancer. Dis. Model Mech. 4(2), 165-178 (2011).

10. Jain RK. Vascular and interstitial barriers to delivery of therapeutic agents in tumors. Cancer Metastasis Rev. 9(3), $253-266$ (1990).

11. Heldin C-H, Rubin K, Pietras K, Ostman A. High interstitial fluid pressure - an obstacle in cancer therapy. Nat. Rev. Cancer 4(10), 806-813 (2004).

12. Curti BD. Physical barriers to drug delivery in tumors. Crit. Rev. Oncol. Hematol. 14, 29-39 (1993).

13. Wright AA, Cronin A, Milne DE et al. Use and effectiveness of intraperitoneal chemotherapy for treatment of ovarian cancer. J. Clin. Oncol. 33(26), 2841-2847 (2015).

14. Wu Y, Zhou L, Lu Y. Intrathecal chemotherapy as a treatment for leptomeningeal metastasis of non-small cell lung cancer: a pooled analysis. Oncol. Lett. 12(2), 1301-1314 (2016).

15. Ko Y, Karanicolas PJ. Hepatic arterial infusion pump chemotherapy for colorectal liver metastases: an old technology in a new era. Curr. Oncol. 21(1), 116-121 (2014).

16. Karanicolas PJ, Metrakos $\mathrm{P}$, Chan $\mathrm{K}$ et al. Hepatic arterial infusion pump chemotherapy in the management of colorectal liver metastases: expert consensus statement. Curr. Oncol. 21(1), e129-e136 (2014).

17. Dizon DS, Schwartz J, Kemeny N. Regional chemotherapy: a focus on hepatic artery infusion for colorectal cancer liver metastases. Surg. Oncol. Clin. N. Am. 17(4), 759-771, viii (2008).

18. Chen HS, Gross JF. Intra-arterial infusion of anticancer drugs: theoretic aspects of drug delivery and review of responses. Cancer Treat. Rep. 64(1), 31-40 (1980).

19. Collins JM. Pharmacologic rationale for regional drug delivery. J. Clin. Oncol. 2(5), 498-504 (1984).

20. Ensminger WD. Intrahepatic arterial infusion of chemotherapy: pharmacologic principles. Semin. Oncol. 29(2), 119-125 (2002). 
21. Farouil G, Deschamps F, Barah A et al. Interventional revisions of malfunctions affecting surgically implanted port-catheters for hepatic artery infusion. Surg. Oncol. 22(1), 48-54 (2013).

22. Kanat $\mathrm{O}$, Gewirtz A, Kemeny $\mathrm{N}$. What is the potential role of hepatic arterial infusion chemo-therapy in the current armamentorium against colorectal cancer. J. Gastrointest. Oncol. 3(2), 130-138 (2012).

23. Rammohan A, Sathyanesan J, Ramaswami S et al. Embolization of liver tumors: past, present and future. World J. Radiol. 4(9), 405-412 (2012).

24. Vogl TJ, Gruber T, Balzer JO, Eichler K, Hammerstingl R, Zangos S. Repeated transarterial chemoembolization in the treatment of liver metastases of colorectal cancer: prospective study. Radiology 250(1), 281-289 (2009).

25. Varela M, Real MI, Burrel M et al. Chemoembolization of hepatocellular carcinoma with drug eluting beads: efficacy and doxorubicin pharmacokinetics. J. Hepatol. 46(3), 474-481 (2007).

26. Poon RTP, Tso WK, Pang RW et al. A Phase I/II trial of chemoembolization for hepatocellular carcinoma using a novel intra-arterial drug-eluting bead. Clin. Gastroenterol. Hepatol. 5(9), 1100-1108 (2007).

27. Malagari K, Chatzimichael K, Alexopoulou E et al. Transarterial chemoembolization of unresectable hepatocellular carcinoma with drug eluting beads: results of an open-label study of 62 patients. Cardiovasc. Intervent. Radiol. 31(2), 269-280 (2008).

28. Martin R, Geller D, Espat J et al. Safety and efficacy of trans arterial chemoembolization with drug-eluting beads in hepatocellular cancer: a systematic review. Hepatogastroenterology 59(113), 255-260 (2012).

- Describes the treatment response, survival and safety of transcatheter arterial chemoembolization used with drug-eluting beads for the treatment of hepatocellular carcinoma and discusses future therapeutic possibilities.

29. Lewis AL, Gonzalez MV, Lloyd AW et al. DC bead: in vitro characterization of a drug-delivery device for transarterial chemoembolization. J. Vasc. Interv. Radiol. 17(2 Pt 1), 335-342 (2006).

30. Lewis AL, Gonzalez MV, Leppard SW et al. Doxorubicin eluting beads - 1: effects of drug loading on bead characteristics and drug distribution. J. Mater. Sci. Mater. Med. 18(9), 1691-1699 (2007).

31. Lammer J, Malagari K, Vogl T et al. Prospective randomized study of doxorubicin-eluting-bead embolization in the treatment of hepatocellular carcinoma: results of the PRECISION V study. Cardiovasc. Intervent. Radiol. 33(1), 41-52 (2010).

32. Lin M-T, Kuo P-H. Pulmonary lipiodol embolism after transcatheter arterial chemoembolization for hepatocellular carcinoma. JRSM Short Rep. 1(1), 6 (2010).

33. Mei Q, Yanhao L. Transcatheter arterial embolization of hepatic arteriovenous shunts in patients with hepatocellular carcinoma. Semin. Interv. Radiol. 29(3), 237-240 (2012).

34. Takayasu K, Arii S, Sakamoto M et al. Clinical implication of hypovascular hepatocellular carcinoma studied in 4,474 patients with solitary tumour equal or less than $3 \mathrm{~cm}$. Liver Int. 33(5), 762-770 (2013).

35. Guadagni S, Aigner KR, Palumbo G et al. Pharmacokinetics of mitomycin C in pelvic stopflow infusion and hypoxic pelvic perfusion with and without hemofiltration: a pilot study of patients with recurrent unresectable rectal cancer. J. Clin. Pharmacol. 38(10), 936-944 (1998).

36. Hsiao JH, Tsai CC, Liang TJ. Adjuvant hepatic arterial infusion chemotherapy is beneficial for selective patients with hepatocellular carcinoma undergoing surgical treatment. Int. J. Surg. 45, 35-41 (2017).

37. Davis J, Pandalai P, Ripley TR et al. Regional chemotherapy in locally advanced pancreatic cancer: RECLAP Trial. Trials 12, 129 (2011).

38. Meyer F, Gebauer T, Grote R et al. Results of regional chemotherapy using the aortic stop-flow technique in advanced pancreatic carcinoma. Surg. Today 36(2), 155-161 (2006).

39. Teicher BA, Lazo JS, Sartorelli AC. Classification of antineoplastic agents by their selective toxicities toward oxygenated and hypoxic tumor cells. Cancer Res. 41(1), 73-81 (1981).

40. Vaupel P, Kallinowski F, Okunieff P. Blood flow, oxygen and nutrient supply, and metabolic microenvironment of human tumors: a review. Cancer Res. 49(23), 6449-6465 (1989).

41. Rockwell S. Use of hypoxia-directed drugs in the therapy of solid tumors. Semin. Oncol. 19(4 Suppl. 11), 29-40 (1992).

42. Aigner KR, Gailhofer S. Celiac axis infusion (MMC, CDDP, 5-FU) and aortic stop flow infusion vs abdominal hypoxic perfusion (MMC) in UICC stage III/IV pancreatic cancer: 48 patients. Reg. Cancer Treat. 6(Suppl. 1), 3 (1993).

43. Fiorentini G, Poddie D, Ricci S et al. Intra-aortic stop-flow infusion (IASFI) with hypoxic abdominal perfusion (HAP) in UICC stage III/IV pancreatic carcinoma (PC): report of a Phase II study. Reg. Cancer Treat. 9(2), 88-91 (1996).

44. Lorenz M, Petrowsky H, Heinrich S et al. Isolated hypoxic perfusion with mitomycin C in patients with advanced pancreatic cancer. Eur. J. Surg. Oncol. 24(6), 542-547 (1998).

45. van IJken MGA, van Etten B, Guetens G et al. Balloon catheter hypoxic abdominal perfusion with mitomycin C and melphalan for locally advanced pancreatic cancer: a Phase I-II trial. Eur. J. Surg. Oncol. 30(6), 671-680 (2004).

46. Skarsgard LD, Skwarchuk MW, Vinczan A, Kristl J, Chaplin DJ. The cytotoxicity of melphalan and its relationship to pH, hypoxia and drug uptake. Anticancer Res. 15(1), 219-223 (1995). 
47. Guadagni S, Clementi M, Valenti M et al. Hypoxic abdominal stop-flow perfusion in the treatment of advanced pancreatic cancer: a Phase II evaluation/trial. Eur. J. Surg. Oncol. 33(1), 72-78 (2007).

48. de Bree E, Romanos J, Tsogkas J et al. Complications and toxicity after abdominal and pelvic hypoxic stop-flow perfusion chemotherapy: incidence and assessment of risk factors. Ann. Surg. Oncol. 19(11), 3591-3597 (2012).

49. Reddy S, Kesmodel S, Alexander HR Jr. Isolated hepatic perfusion for patients with liver metastases. Ther. Adv. Med. Oncol. 6(4), 180-194 (2014).

- In cases of isolated liver, metastases surgical resection offers the best long-term survival. However, up to $80 \%$ of patients with liver metastases have unresectable disease due to combinations of excessive tumor burden, anticipated insufficient liver remnant with intact vascular inflow/outflow and biliary drainage or medical comorbidity. Clearly alternative therapies are needed. This review describes the development of intrahepatic perfusion, initial clinical results, open and percutaneous intrahepatic perfusion techniques and contemporary long-term treatment outcomes.

50. Alexander HR Jr, Libutti SK, Bartlett DL, Puhlmann M, Fraker DL, Bachenheimer LC. A Phase I-II study of isolated hepatic perfusion using melphalan with or without tumor necrosis factor for patients with ocular melanoma metastatic to liver. Clin. Cancer Res. 6(8), 3062-3070 (2000).

51. Jones A, Alexander HR Jr. Development of isolated hepatic perfusion for patients who have unresectable hepatic malignancies. Surg. Oncol. Clin. N. Am. 17(4), 857-876 (2008).

52. Burgmans MC, de Leede EM, Martini CH. Percutaneous isolated hepatic perfusion for the treatment of unresectable liver malignancies. Cardiovasc. Interv. Radiol. 39, 801-804 (2016).

- Discusses the current indications, technique and patient management of percutaneous isolated hepatic perfusion and provides an overview of the available data.

53. Alexander HR Jr, Libutti SK, Pingpank JF et al. Hyperthermic isolated hepatic perfusion using melphalan for patients with ocular melanoma metastatic to liver. Clin. Cancer Res. 9(17), 6343-6349 (2003).

54. Forster MR, Rashid OM, Perez MC et al. Chemosaturation with percutaneous hepatic perfusion for unresectable metastatic melanoma or sarcoma to the liver: a single institution experience. J. Surg. Oncol. 109(5), 434-439 (2014).

55. Noter SL, Rothbarth J, Pijl ME et al. Isolated hepatic perfusion with high-dose melphalan for the treatment of uveal melanoma metastases confined to the liver. Melanoma Res. 14(1), 67-72 (2004).

56. Agarwala SS, Eggermont AMM, O’Day S, Zager JS. Metastatic melanoma to the liver: a contemporary and comprehensive review of surgical, systemic, and regional therapeutic options. Cancer 120(6), 781-789 (2014).

57. Rizell M, Mattson J, Cahlin C, Hafstrom L, Lindner P, Olausson M. Isolated hepatic perfusion for liver metastases of malignant melanoma. Melanoma Res. 18(2), 120-126 (2008).

58. Miao N, Pingpank JF, Alexander HR, Steinberg SM, Beresneva T, Quezado ZMN. Percutaneous hepatic perfusion in patients with metastatic liver cancer: anesthetic, hemodynamic, and metabolic considerations. Ann. Surg. Oncol. 15(3), 815-823 (2008).

59. Lillemoe HA, Alexander HR. Current status of percutaneous hepatic perfusion as regional treatment for patients with unresectable hepatic metastases: a review. Am. Oncol. Hematol. Rev. 15-23 (2014).

60. Hughes MS, Zager J, Faries M et al. Results of a randomized controlled multicenter Phase III trial of percutaneous hepatic perfusion compared with best available care for patients with melanoma liver metastases. Ann. Surg. Oncol. 23(4), 1309-1319 (2016).

61. Fick A. On liquid diffusion. J. Memb. Sci. 100, 33-38 (1995).

62. Whitaker S. Flow in porous media I: a theoretical derivation of Darcy's Law. Transp. Porous Media 1, 3-25 (1986).

63. Aigner KR, Stephens FO. Induction Chemotherapy - Integrated Treatment Programs for Locally Advanced Cancers. Springer, Berlin, Germany (2011).

64. Gilbert-Kawai E, Wittenberg M. Essential Equations for Anaesthesia: Key Clinical Concepts for the FRCA and EDA. Cambridge University Press, Cambridge, UK (2014).

65. Woodcock TE, Woodcock TM. Revised Starling equation and the glycocalyx model of transvascular fluid exchange: an improved paradigm for prescribing intravenous fluid therapy. Br. J. Anaesth. 108(3), 384-394 (2012).

66. López-Magañna JA, Richards HK, Radolovich DK et al. Critical closing pressure: comparison of three methods. J. Cereb. Blood Flow Metab. 29(5), 987-993 (2009).

67. Priya W, Buja LM. Oncosis: an important non-apoptotic mode of cell death. Exp. Mol. Pathol. 93(3), 302-308 (2012).

68. Khin NY, Dijkstra ML, Huckson M et al. Hypertensive extracorporeal limb perfusion for critical limb ischemia. J. Vasc. Surg. 58(5), $1244-1253$ (2013).

69. Hoekstra HJ. Extremity perfusion for sarcoma. Surg. Oncol. Clin. N. Am. 17(4), 805-824 (2008).

70. Grunhagen DJ, de Wilt JHW, van Geel AN, Eggermont AMM. Isolated limb perfusion for melanoma patients - a review of its indications and the role of tumour necrosis factor-alpha. Eur. J. Surg. Oncol. 32(4), 371-380 (2006).

71. Lane RJ, Phillips M, McMillan D, Huckson M, Liang SW-U, Cuzzilla M. Hypertensive extracorporeal limb perfusion (HELP): a new technique for managing critical lower limb ischemia. J. Vasc. Surg. 48(5), 1156-1165; discussion 1165 (2008). 
- Reports on the results in humans of hypertensive extracorporeal limb perfusion technology in the prevention of major limb amputation due to ischemia. The same basic hardware was modified to hyperperfuse solid tumors with chemotherapy.

72. Kam PCA, Thompson JF. Pharmacokinetics of regional therapy: isolated limb infusion and other low flow techniques for extremity melanoma. Surg. Oncol. Clin. N. Am. 17(4), 795-804 (2008).

73. Lane RJ, Khin NY, Rogan CM et al. Safety and feasibility of repeatable hepatic vascular isolation chemotherapy: a pilot study. Ann. Surg. Oncol. 23(11), 3699-3708 (2016).

-. Presents a novel method for repeatable organ isolation via an implantable multicatheter access system to administer selective intra-arterial chemotherapy. The described repeatable vascular isolation protocol optimizes endothelial permeability, bulk fluid flow, the hydraulic and oncotic forces thereby addressing the multiple 3D spatiotemporal barriers to current chemotherapy delivery.

74. Katz SC, Burga RA, McCormack E et al. Phase I hepatic immunotherapy for metastases study of intra-arterial chimeric antigen receptor-modified T-cell therapy for CEA+ liver metastases. Clin. Cancer Res. 21(14), 3149-3159 (2015).

75. Graham MA, Lockwood GF, Greenslade D, Brienza S, Bayssas M, Gamelin E. Clinical pharmacokinetics of oxaliplatin: a critical review. Clin. Cancer Res. 6(4), 1205-1218 (2000).

76. Eipel C, Abshagen K, Vollmar B. Regulation of hepatic blood flow: the hepatic arterial buffer response revisited. World J. Gastroenterol. 16(48), 6046-6057 (2010).

77. Takimoto $\mathrm{CH}$, Remick SC, Sharma S et al. Administration of oxaliplatin to patients with renal dysfunction: a preliminary report of the national cancer institute organ dysfunction working group. Semin. Oncol. 4(Suppl. 15), 20-25 (2003).

78. Rietbroek RC, van de Vaart PJ, Haveman J et al. Hyperthermia enhances the cytotoxicity and platinum-DNA adduct formation of lobaplatin and oxaliplatin in cultured SW 1573 cells. J. Cancer Res. Clin. Oncol. 123(1), 6-12 (1997).

79. Stephens FO. Why use regional chemotherapy? Principles and pharmacokinetics. Reg. Cancer Treat. 1, 4-10 (1988).

80. Stephens FO. Intraarterial induction chemotherapy in locally advanced stage III breast cancer. Cancer 66(4), 645-650 (1990).

- Management of locally advanced Stage III primary breast cancer remains a formidable problem. Surgical excision has resulted in a high incidence of local wound recurrence, and radiotherapy alone has failed to control local disease. The paper presents the possibility of using directed arterial chemotherapy to the effected breast in order to induce changes to make it more amenable to standard treatment.

81. Loi S, Sirtaine N, Piette F et al. Prognostic and predictive value of tumor-infiltrating lymphocytes in a Phase III randomized adjuvant breast cancer trial in node-positive breast cancer comparing the addition of docetaxel to doxorubicin with doxorubicin-based chemotherapy: BIG 02-98. J. Clin. Oncol. 31(7), 860-867 (2013).

82. Salgado R, Denkert C, Campbell C et al. Tumor-infiltrating lymphocytes and associations with pathological complete response and event-free survival in HER2-positive early-stage breast cancer treated with lapatinib and trastuzumab: a secondary analysis of the NeoALTTO trial. JAMA Oncol. 1(4), 448-454 (2015).

83. Zhang L, Conejo-Garcia JR, Katsaros D et al. Intratumoral T cells, recurrence, and survival in epithelial ovarian cancer. N. Engl. J. Med. 348(3), 203-213 (2003).

84. Cogdill AP, Andrews MC, Wargo JA. Hallmarks of response to immune checkpoint blockade. Br. J. Cancer 117(1), 1-7 (2017).

-• Recent advances have been made in the treatment of cancer through the use of immune checkpoint blockade, with approval of several checkpoint blockade regimens spanning multiple cancer types. The paper outlines hallmarks of response to immune checkpoint blockade, with a discussion of their relevance to immune monitoring and mechanisms of resistance.

85. Schuster DM, Nanni C, Fanti S et al. Anti-1-amino-3-18F-fluorocyclobutane-1-carboxylic acid: physiologic uptake patterns, incidental findings, and variants that may simulate disease. J. Nucl. Med. 55(12), 1986-1992 (2014).

86. Oka S, Okudaira H, Yoshida Y, Schuster DM, Goodman MM, Shirakami Y. Transport mechanisms of trans-1-amino-3-fluoro[1-(14)C]cyclobutanecarboxylic acid in prostate cancer cells. Nucl. Med. Biol. 39(1), 109-119 (2012).

87. Young S, Rosenberg M, Shrestha P, Golzarian J. Prostate artery embolization techniques. Endovasc. Today 16(4), 57-60 (2017). 
